\title{
Structural Analysis and Optimization of Liquid Nitrogen Fire Monitor Based on FLUENT
}

\author{
Yunxing Hou ${ }^{1}$, Xiaowei Yang ${ }^{1}$, Fangyan Ren ${ }^{1}$ and Yanjun Liu ${ }^{1,2,3, *}$ \\ ${ }^{1}$ Institute of Marine Science and Technology, Shandong University, Qingdao 266237, Shandong, China \\ ${ }^{2}$ School of Mechanical Engineering, Shandong University, Jinan 250061, Shandong, China \\ ${ }^{3}$ Key Laboratory of High Efficiency and Clean Mechanical Manufacture, Shandong University, Jinan 250061, Shandong, China \\ ${ }^{*}$ Corresponding author
}

\begin{abstract}
The nozzle is the key component to transform the fluid potential energy into kinetic energy. It is of great significance to the operation the fire protection system. A simple two-dimensional structure of the nozzle is developed. The outlet velocity of the nozzle model is calculated according to hydrodynamics theory. The liquid nitrogen jet performance of the nozzle is simulated by using the FLUENT software. The simulation results are consistent with the theoretical calculations. Then the effect of the rectifier tube and the shrinkage angle was studied. The results show that the rectifier tube can increase the liquid nitrogen jet's velocity. A larger shrinkage angle can get a higher velocity. Finally, it is proved that a local negative pressure can be eliminated by adding transition fillet.
\end{abstract}

Keywords—liquid nitrogen fire monitor; numerical simulation; nozzle

\section{INTRODUCTION}

Fire is one of the most normal disasters in natural and social disasters, causing serious harm to human life and property. In 2016, the direct economic losses caused by fires is more than 3.72 billion yuan. At the same time, with the rapid development of the economy and the improvement of people's living standards, the tall buildings are more intense than before. It also increased the difficulty to extinguish a fire, which is more likely to cause huge social and economic losses.

At present, fire water cannon is widely used in the practice for the reason of small size, long range, light weight and so on [1]. For some important places, water, foam extinguishing agent can effectively extinguish the fire, but they will inevitably damage the goods. Liquid nitrogen fire extinguisher is pollution-free, water saving, and no water stains, etc. And liquid nitrogen fire-fighting equipment has been developed abroad [2]. In the past, the research on fire cannons in China was based on water jet fluid and rarely involved in liquid nitrogen. Although some scholars have studied the liquid nitrogen fire-fighting equipment, it is also limited to the field experiment, and the fire extinguishing characteristics are obtained only from the subjective view [3][4]. In this paper, the simulation analysis of the liquid nitrogen fire monitor is carried out, and the influence of its structural parameters on the performance is analyzed, which provides a reference for the design of the liquid nitrogen fire monitor nozzle.

\section{The Working PrinciPle OF LIQUid Nitrogen Fire- FIGHTING EQUIPMENT}

First, liquid nitrogen fire-fighting equipment is a new type of special fire-fighting equipment. It can not only fire liquid nitrogen alone but also use the enormous energy generated by gasification and expansion of liquid nitrogen to push water into fine water mist to extinguish the fire. The inner and outer layers of the liquid nitrogen transfer line are made of plastic and metal materials, which are resistant to high and low temperatures. Liquid nitrogen will spray out with the help of fire-fighting nozzle. Liquid nitrogen vaporizes instantaneously, and the volume rapidly expands to generate a large amount of nitrogen, which floods the entire combustion space, causing a significant decrease in oxygen content. When the volume fraction of oxygen drops below $15 \%$, the combustion is terminated [5]. At the same time, liquid nitrogen can absorb a large amount of heat generated by the combustion in the process of gasification, so that the temperature of the combustible material is rapidly reduced to below its ignitable point, and the fire is extinguished.

\section{ThEORETICAL CALCULATION}

According to the structure, Fire-fighting nozzle can be divided into full water column nozzle and deflector-type nozzle. Between them, the full water column nozzle is widely used in fire-fighting for the simple structure and stable performance. In this paper, the full water column nozzle is studied, and the twodimensional structure of the nozzle is simplified as shown in Figure I.

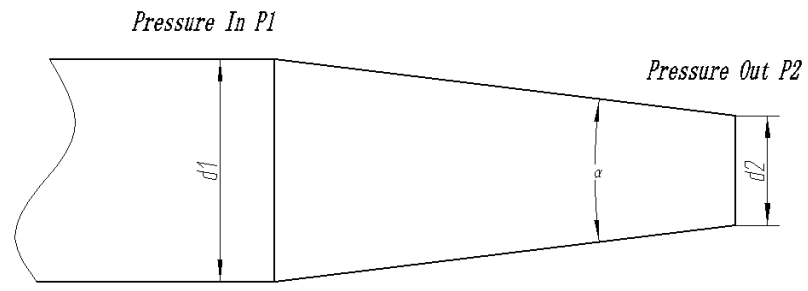

FIGURE I. STRUCTURE OF THE NOZZLE

The pipe diameter of fire monitor $d_{1}$ is $40 \mathrm{~mm}$, the nozzle shrinkage angle $\alpha$ is $14^{\circ}$ and the outlet diameter $d_{2}$ is $10 \mathrm{~mm}$. 
In ideal condition, the height difference between inlet and outlet and the loss in flow process are ignored, and the Bernoulli equation [7] has been used as follows:

$$
p_{1}+\frac{1}{2} \rho_{1} v_{1}^{2}=p_{2}+\frac{1}{2} \rho_{2} v_{2}^{2}
$$

where $p_{1}, p_{2}, v_{1}, v_{2}$ represent the pressure and velocity of liquid nitrogen at the inlet and outlet of the nozzle, respectively.

Between the two points of the pressure inlet and outlet, the equation of continuity is applied in the following equation:

$$
A_{1} v_{1}=A_{2} v_{2}
$$

In the equation, $A_{1}$ and $A_{2}$ respectively represent the area of the nozzle inlet and outlet.

Assume there is no change in the liquid nitrogen density between the inlet and outlet. By (1), (2), following equation can be obtained:

$$
V_{2}=\sqrt{\frac{2\left(p_{1}-p_{2}\right)}{\rho\left[1-\left(\frac{d_{2}}{d_{1}}\right)^{4}\right]}}
$$

The liquid nitrogen density $\rho$ is $806.08 \mathrm{~kg} / \mathrm{m}^{3}$, the inlet pressure is $2.0 \mathrm{MPa}$, and the outlet pressure is the standard atmospheric pressure. Substituting each value into equation (3) yields:

$$
v_{2}=70.6 \mathrm{~m} / \mathrm{s} \text {. }
$$

In the ideal condition, without considering the effects of resistance, etc., the spray pattern of the fire monitor is similar to the ballistic movement [8]. Its theoretical range formula is:

$$
d=v_{2} \bullet \cos \theta \bullet t=\frac{v_{2}^{2} \bullet \sin 2 \theta}{g}
$$

where $d$ represents the range of fluid; $\theta$ represents the angle between fluid spray and horizontal; $t$ is total flight time of fluid; $g$ represents the acceleration of gravity.
From (4), it can be obtained that when $\theta=\frac{\pi}{4}$ has the maximum range. So in order to get a farther range when $\theta$ is determined, the outlet velocity of the nozzle must be increased.

\section{MODEL ANALYSIS}

The research object of this paper is high-pressure liquid nitrogen jet, its flow field is single-phase flow. Assume that the fluid is incompressible, and heat transfer is not considered in this transient model. For liquid nitrogen fire-fighting monitor, the change rate of velocity and pressure during the movement in the pipe is large, resulting in an unstable state, which makes the jet motion of liquid nitrogen fire monitor a turbulent jet. The standard k- $\varepsilon$ model is a widely used two-equation model, which introduces the equations for the turbulent kinetic energy $\mathrm{k}$ and its rate of dissipation $\varepsilon$ respectively [9]. The equations are as follows:

$$
\begin{aligned}
& \frac{\partial}{\partial t}(p k)+\frac{\partial}{\partial x_{i}}\left(p k u_{i}\right)=\frac{\partial}{\partial x_{j}}\left[\left(\mu+\frac{\mu_{t}}{\sigma_{k}}\right) \frac{\partial k}{\partial x_{j}}\right]+G_{k}+G_{b}- \\
& \rho \varepsilon-Y_{M}+S_{k}
\end{aligned}
$$

and

$$
\begin{aligned}
& \frac{\partial}{\partial t}(\rho \varepsilon)+\frac{\partial}{\partial x_{i}}\left(\rho \varepsilon u_{i}\right)=\frac{\partial}{\partial x_{j}}\left[\left(\mu+\frac{\mu_{t}}{\sigma_{\varepsilon}}\right) \frac{\partial \varepsilon}{\partial x_{j}}\right]+ \\
& C_{1 \varepsilon} \frac{\varepsilon}{k}\left(G_{k}+C_{3 \varepsilon} G_{b}\right)-C_{2 \varepsilon} \rho \frac{\varepsilon^{2}}{k}+S_{\varepsilon}
\end{aligned}
$$

where, Gk represents the generation of turbulence kinetic energy due to the mean velocity gradients. $\mathrm{Gb}$ is the generation of turbulence kinetic energy due to buoyancy. $\mathrm{Y}_{\mathrm{M}}$ represents the contribution of the fluctuating dilatation in compressible turbulence to the overall dissipation rate. $\mathrm{C}_{1 \varepsilon}, \mathrm{C}_{2 \varepsilon}$ and $\mathrm{C}_{3 \varepsilon}$ are constants. $\sigma_{\mathrm{k}}, \sigma_{\varepsilon}$ are the turbulent Prandtl numbers for $\mathrm{k}$ and $\varepsilon$, respectively. $S_{k}$ and $S_{\varepsilon}$ are user-defined source terms.

The numerical model used in this paper is the standard k- $\varepsilon$ model. The standard k- $\varepsilon$ model belongs to the high Reynolds number model, and the accuracy of its calculation is affected by the flow state of the research object, which is suitable for the fully developed turbulent flow field calculation. For the simulation calculation of liquid nitrogen fire monitor, the turbulence in the boundary layer area is not fully developed, and its Reynolds number is low, so the wall area is calculated using the low Reynolds number k- $\varepsilon$ model.

Based on the $\mathrm{k}-\varepsilon$ model of the above analysis, simultaneous mass conservation equations, momentum conservation equations, energy conservation equations, and $\mathrm{k}-\varepsilon$ equations can solve the problem of liquid nitrogen fire monitor flow rate. 


\section{GEOMETRIC MODEL}

Considering that the fire monitor model is an axisymmetric figure, the geometric model adopts a two-dimensional symmetry model, which can reduce the calculation amount and accelerate the convergence speed [10]. According to the twodimensional structure model of the nozzle, the inlet $\mathrm{AB}$ of the simulation model is $20 \mathrm{~mm}$, the outlet $\mathrm{CD}$ is $5 \mathrm{~mm}$, and the shrinkage angle is $14^{\circ}$. Software ICEM CFD was used for meshing. In the meshing process, the mesh near the wall surface is refined for higher accuracy. The unstructured meshing method is used in the research process. At the same time, the mesh independence is verified. The mesh result is shown in Figure II.

ANSYS

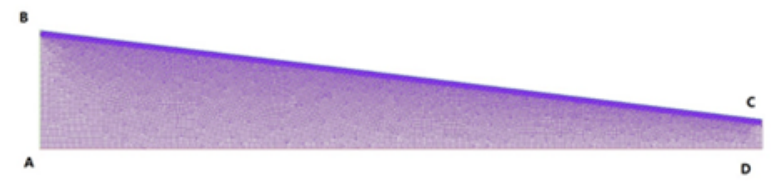

FIGURE II. MESHING

The turbulence model is defined in Fluent. The turbulence model adopts the standard $k-\varepsilon$ model without heat exchange. The boundary conditions are set up as follows, set $\mathrm{AB}$ as pressure inlet, $\mathrm{CD}$ as pressure outlet, $\mathrm{BC}$ as no slip wall, and $\mathrm{AD}$ as the symmetry axis. Liquid nitrogen is selected for the fluid and the default values are used for each parameter. The SIMPLE algorithm is used to solve the coupling of pressure field and velocity field, and the relaxation factor takes the default value.

\section{Simulation RESUlts AND ANALYSIS}

\section{A. Velocity and Pressure Analysis}

Based on the above model analysis and boundary conditions, the liquid nitrogen fire monitor is carried out to obtain the contours such as pressure distribution and velocity distribution. The change of pressure and velocity is shown in Figure III. Along the direction of the liquid nitrogen fire monitor, the pressure gradually decreases and the speed gradually increases. At the outlet near the nozzle, the gradient of pressure decreases and the velocity increases rapidly. Where the pressure decreases rapidly, the velocity increases faster because the potential energy is converted into kinetic energy, which conforms to the laws of the Bernoulli equation. In the simulation results, the axial velocity of liquid nitrogen at the outlet of the nozzle was $70.5 \mathrm{~m} / \mathrm{s}$. The simulation result is consistent with the theoretical calculations.

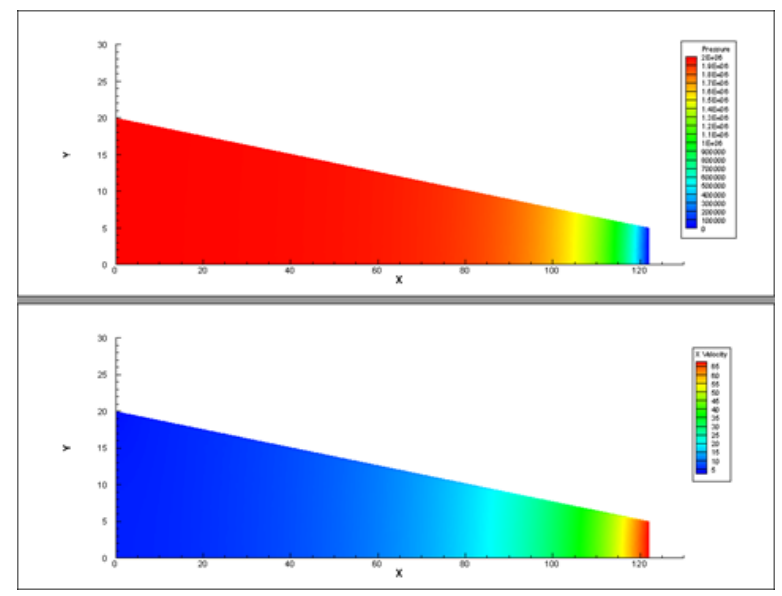

FIGURE III. THE CONTOURS OF PRESSURE AND VELOCITY DISTRIBUTION

The axial velocity change on the symmetry axis $\mathrm{AD}$ is shown in Figure IV. As the cross-sectional area becomes smaller, the velocity of the fluid becomes larger. Near the outlet of the nozzle, the velocity gradient changes greatly. The convergence and stability of liquid nitrogen jet become worse. According to the theory of jet flow, the jet of the nozzle will diverge in the air, and the divergence angle of the jet is equal to the shrinkage angle [11]. If liquid nitrogen is directly sprayed into the air along the conical tube, turbulence will occur due to a large change in cross-sectional area, resulting in energy loss and affecting the range. At the same time, liquid nitrogen fire monitor needs to avoid the human body during application. So, it is necessary to add a cylindrical rectifier tube with length $L$ at the muzzle to obtain a smooth liquid nitrogen jet.

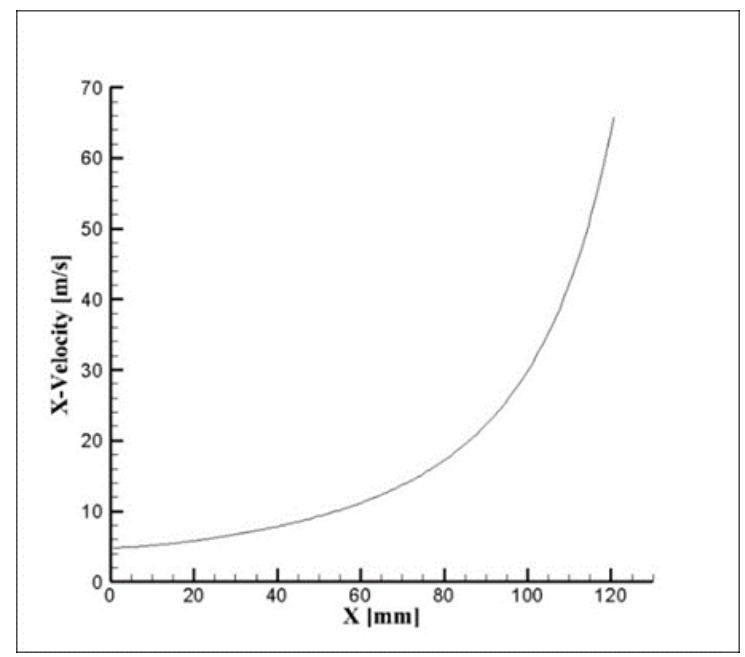

FIGURE IV. THE AXIAL VELOCITY CHANGE ON THE SYMMETRY AXIS AD

\section{B. Rectifier Tube Analysis}

In order to determine the influence of the rectifier tube on the outlet velocity, the influence of the length parameter of the rectifier tube is studied firstly. Figure $\mathrm{V}$ is a comparison of the axial velocity of the rectifier tubes under the same conditions among 6 rectifier tubes with different lengths. Because the 
length of those rectifier tubes various, the length is dealt with dimensionless. As can be seen from Figure V, the rectifier tube can improve the liquid nitrogen jet's velocity. When the length of the rectifier tube is relatively small, the change of the outlet axis velocity is not obvious. This is because when $L$ is small, the turbulent energy of the jet at the outlet of the nozzle is large, the velocity gradient changes greatly, and the rectification effect is not obvious. As the length of the rectifier tube increases, the rectification effect is significant.

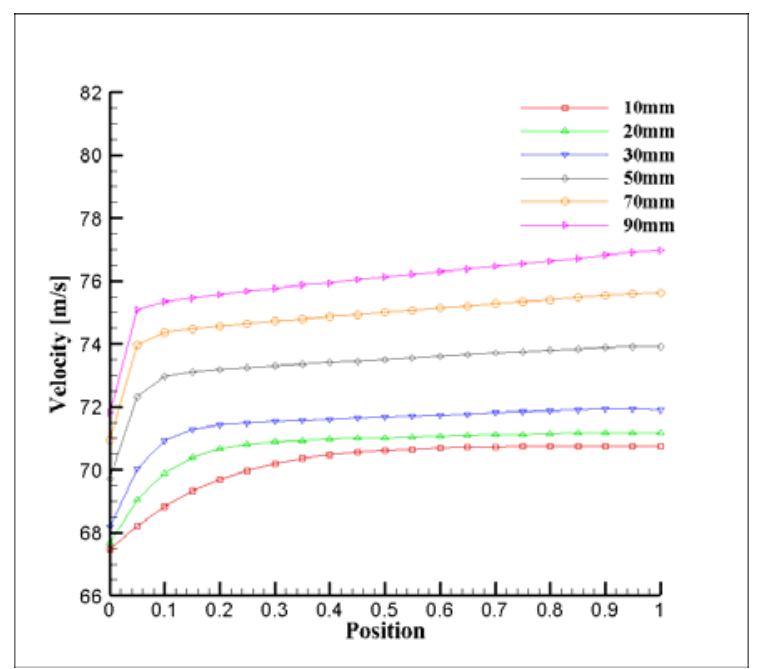

FIGURE V. A COMPARISON OF THE AXIAL VELOCITY OF THE VARIATION OF THE RECTIFIER TUBE

Figure VI shows the variation of the ratio of length to diameter and the outlet axis velocity of three different rectifier tubes. The ratio of length to diameter is 1.5, 2 and 2.5 respectively. The corresponding diameter of each group is $10 \mathrm{~mm}, 14 \mathrm{~mm}, 18 \mathrm{~mm}, 22 \mathrm{~mm}$, and $26 \mathrm{~mm}$ respectively.

From the simulation results in Figure VI, with the increase of the ratio of the rectifier tube, the outlet axis velocity of the liquid nitrogen jet is increasing. For a given aspect ratio, as the diameter of the rectifier tube increases, the outlet velocity of the liquid nitrogen jet increases first and then decreases. This is because the increase in the length of the rectifier tube brings excessive pipeline loss so that the loss is greater than the rectifying effect and affects the range of the liquid nitrogen.

Considering actual fire-fighting operation requirements, the increasing length of the rectifier tube will also increase the size and the consumption of materials. Therefore, for the liquid nitrogen fire monitor studied in this paper, it is appropriate to take the length of the rectification section from $40 \mathrm{~mm}$ to $50 \mathrm{~mm}$, and the length-diameter ratio should be 2.5.

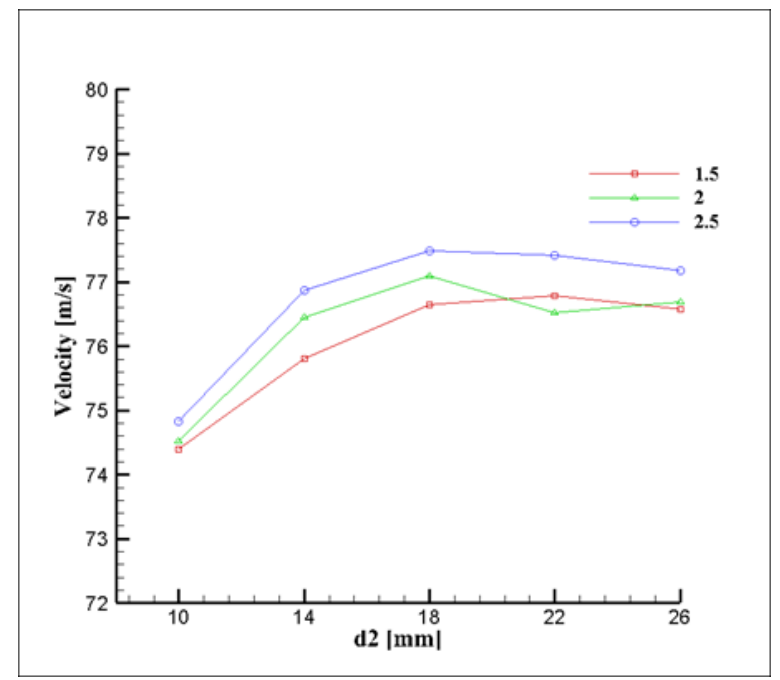

FIGURE VI. A COMPARISON OF THE AXIAL VELOCITY OF THE VARIATION OF THE LENGTH-DIAMETER RATIO

\section{Effect of Shrinkage Angle}

The shrinkage section of the fire monitor is connected with the pipes, and its value affects the performance of the liquid nitrogen jet. This paper simulates the fire monitor at $10^{\circ}, 12^{\circ}$, $14^{\circ}, 16^{\circ}$ and $18^{\circ}$ shrinkage angles. The length of the rectifier tube is $40 \mathrm{~mm}$. Different shrinkage angles correspond to different lengths of the shrinkage section. In order to compare the axial velocity between the different shrinkage angles, the length of the fire monitor is dealt with dimensionless. The simulation results are shown in Figure VII.

It can be seen from Figure VII that the outlet axis velocity of the liquid nitrogen jet decreases as the shrinkage angle increases. The small shrinkage angle leads to an increase in the length of the shrinkage section, which also increases the pipeline loss and reduces the outlet velocity. With larger shrinkage angle, the faster change of the fluid velocity, and the more unstable state of the liquid nitrogen jet, which requires longer rectifier tube.

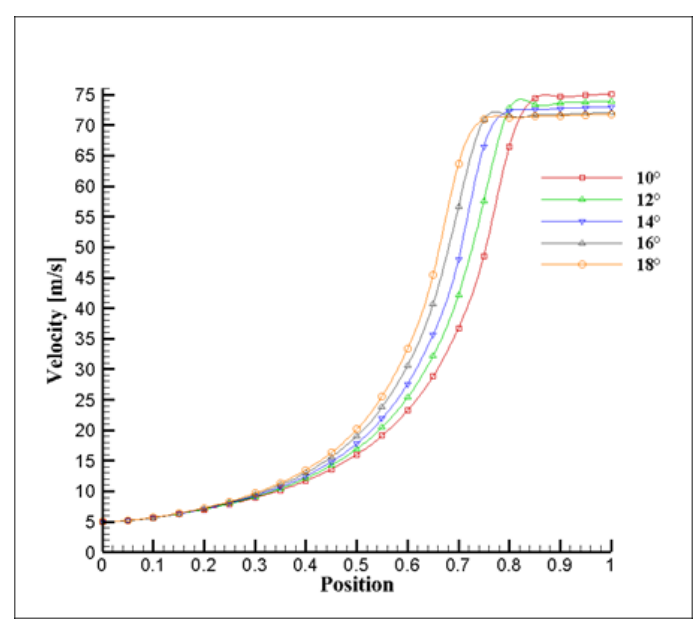

FIGURE VII. A COMPARISON OF THE AXIAL VELOCITY OF THE VARIATION OF THE SHRINKAGE ANGLE 


\section{Structural Optimization}

By observing the pressure contours of the above different simulation results, it can be found that a local negative pressure is generated at the junction of the conical nozzle and the rectifier tube. Especially, when the length of the rectifier tube is small, the local negative pressure range is relatively large. This is due to the large change in the cross-sectional area of the liquid nitrogen jet and the vortex phenomenon [12]. As the length of the rectifier tube increases, the turbulence degree of liquid nitrogen decreases, and the area of local negative pressure decreases, but it is not eliminated. The vortex phenomenon is not conducive to fluid energy conversion and should be eliminated by optimizing the structure. Therefore, structural optimization is performed by adding transition fillets at the junction.

Figure VIII shows the pressure contours comparison at the junction before and after the structural optimization. The above picture is the pressure contour of the $40 \mathrm{~mm}$ rectifier tube with unoptimized structure. The following figure is the pressure contour of the optimized junction under the same structural parameters. From the comparison of Figure VIII, it can be seen that the vortex phenomenon disappears and the liquid nitrogen jet does not produce negative pressure after the transition fillet is added.

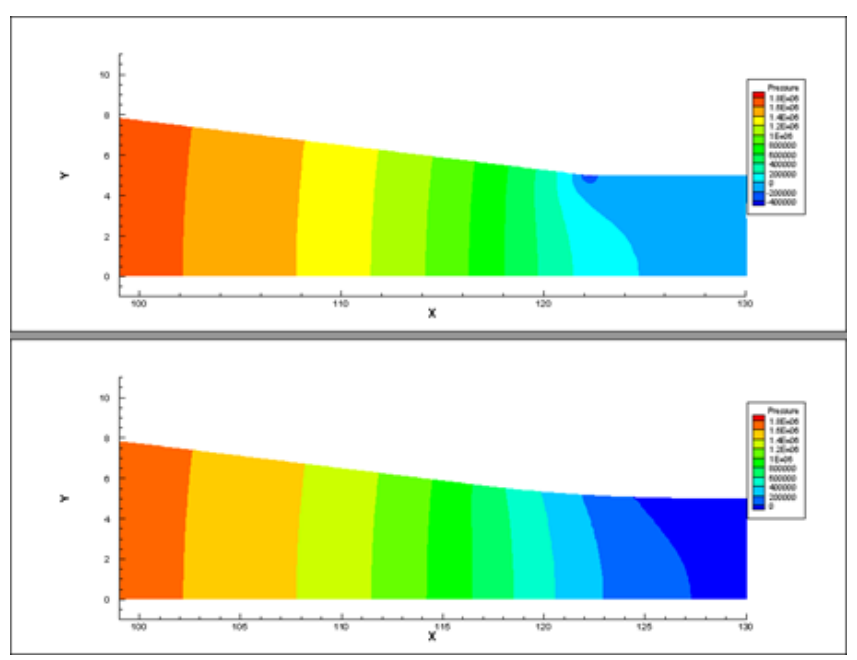

FIGURE VIII. PRESSURE COMPARISON

\section{CONCLUSION}

(1) The rate change of the liquid nitrogen jet velocity in the conical tube increases, which inevitably causes hydraulic loss and affects the range of liquid nitrogen jet. The rectifier tube can improve the unstable state of the liquid nitrogen jet so that the liquid nitrogen exhibits a stable state at the outlet velocity. Liquid nitrogen fire monitor's rectifier tube length is best to take between $40 \sim 50 \mathrm{~mm}$.

(2) The outlet velocity of the fire monitor decreases with the increase of the shrinkage angle. If the shrinkage angle is too small, the length of the shrinkage section will increase, which will increase the production cost.
(3) A negative pressure region will be produced at the junction of the conical nozzle and the rectifier tube. By adding transition fillets at the junction, local negative pressure can be eliminated and the loss of energy conversion can be reduced.

\section{REFERENCES}

[1] W. G. Chen, "The structure paramaters optimization and hydraulic performance reserch of fixed fire water monitor," East China Jiaotong University, 2009.

[2] Y. A. Levendis, M. A. Delichatsios, "Cryogenic suppression of liquid lool fires and wooden crib fires," Suppression, Detecion and Signaling Research and Applications Technical Working Conference 2011, Florida, 2011.

[3] K. X. Ma, X. W. Xong, H. Zhang and C. P. Bean, "Study on the liquefied nitrogen fire fighting strategy," Fire Science and Technology, vol. 4, 2002, pp. 13-14.

[4] H. Zhang, P. Fu, K. X. Ma, and F. Q. Dai, "The liquid nitrogen extinguish fire the device and extinguishes fire the function experiment analysis," Fire Science and Technology, vol. 4, 2003, pp. 303-304.

[5] J. H. Li, "Research and application of liquid nitrogen fire extinguishing technology," The annual meeting of the Chinese People's Armed Police Force Academy and fire fighting and rescue technology forum, Langfang, 2005.

[6] C. Zhu, "Research \& Manufacture on multi-funtion automatic pressure control fire-fighting nozzle and fire cuppression experiment with watermist," Zhejiang University, 2005.

[7] W. Graebel, Engineering Fluid Mechanics: Taylor \& Francis, 2001,pp:169-170.

[8] F. Wang, X. Y. Chen, Y. L. Min, W. M. Wang, " Fitting equation of the fire monitor's jet track", Fire Science and Technology, vol. 6, 2007, pp:656-658.

[9] Z. C. Xie, "Hydraulic properties reserach based on the CFD of fire water cannon," Harbin Engineering University, 2012.

[10] R. P. Bajpai, U. Chandrasekhar, Innovative Design and Development Practices in Aerospace and Automotive Engineering, Springer, 2016, pp:401-403.

[11] R. Shi, "Performance optimization of a long range fire water cannon on CFD Technology," Jiangsu UNivesrity, 2017.

[12] B. Gao, Z. M. Kou, "Mechanism research of negative pressure produced for jet device based on Fluent", Coal Mine Machinery, vol. 7, 2012, pp:77-79. 